\title{
On the Dynamic Nature of the Engram: Evidence for Circuit-Level Reorganization of Object Memory Traces following Reactivation
}

\author{
Boyer D. Winters, Mark C. Tucci, Derek L. Jacklin, James M. Reid, and James Newsome \\ Department of Psychology and Collaborative Neuroscience Program, University of Guelph, Guelph, Ontario N1G 2W1, Canada
}

Research has implicated the perirhinal cortex (PRh) in several aspects of object recognition memory. The specific role of the hippocampus (HPC) remains controversial, but its involvement in object recognition may pertain to processing contextual information in relation to objects rather than object representation per se. Here we investigated the roles of the PRh and HPC in object memory reconsolidation using the spontaneous object recognition task for rats. Intra-PRh infusions of the protein synthesis inhibitor anisomycin immediately following memory reactivation prevented object memory reconsolidation. Similar deficits were observed when a novel object or a salient contextual change was introduced during the reactivation phase. Intra-HPC infusions of anisomycin, however, blocked object memory reconsolidation only when a contextual change was introduced during reactivation. Moreover, disrupting functional interaction between the HPC and PRh by infusing anisomycin unilaterally into each structure in opposite hemispheres also impaired reconsolidation when reactivation was done in an altered context. These results show for the first time that the PRh is critical for reconsolidation of object memory traces and provide insight into the dynamic process of object memory storage; the selective requirement for hippocampal involvement following reactivation in an altered context suggests a substantial circuit level object trace reorganization whereby an initially PRh-dependent object memory becomes reliant on both the HPC and PRh and their interaction. Such trace reorganization may play a central role in reconsolidation-mediated memory updating and could represent an important aspect of lingering consolidation processes proposed to underlie long-term memory modulation and stabilization.

\section{Introduction}

When information is acquired it must undergo a process of consolidation to be retained in long-term memory (McGaugh, 2000; Dudai, 2004). Moreover, when a memory is reactivated, its trace can again become labile and sensitive to disruption. To persist, this labile memory must be reconsolidated. The process of postreactivation memory destabilization and reconsolidation has been demonstrated in numerous species and for myriad memory types (Misanin et al., 1968; Sara, 2000; Nader, 2003; Hardt et al., 2010; Alberini, 2011), including object recognition memory (Bozon et al., 2003; Kelly et al., 2003; Akirav and Maroun, 2006; Rossato et al., 2007; Maroun and Akirav, 2008; Lima et al., 2009; Winters et al., 2009; Davis et al., 2010; Romero-Granados et al., 2010).

Research has revealed a central role for the perirhinal cortex (PRh) in object representation (Buckley and Gaffan, 2006; Barker et al., 2007; Bartko et al., 2007; Bussey and Saksida, 2007;

\footnotetext{
Received June 13, 2011; revised 0ct. 2, 2011; accepted Oct. 24, 2011.

Author contributions: B.D.W. designed research; M.C.T., D.L.J., J.M.R., and J.N. performed research; B.D.W. and D.L.J. analyzed data; B.D.W. wrote the paper.

This research was supported by a National Sciences and Engineering Research Council (NSERC) of Canada Discovery Grant to B.D.W., an NSERC undergraduate student research award to M.C.T., and an Ontario Graduate Scholarship to D.L.J.

Correspondence should be addressed to Dr. Boyer D. Winters, Department of Psychology, University of Guelph, Guelph, 0N N1G 2W1, Canada. E-mail: bwinters@uoguelph.ca.

DOI:10.1523/JNEUROSCI.2968-11.2011

Copyright $\odot 2011$ the authors $\quad 0270-6474 / 11 / 3117719-10 \$ 15.00 / 0$
}

McTighe et al., 2010). Indeed, studies with the spontaneous object recognition (SOR) task for rats have implicated PRh in object memory encoding, consolidation, and retrieval (Winters and Bussey, 2005a,b; Barker et al., 2006a,b). The hippocampus (HPC) may also play a role in object recognition, but the specific involvement of this structure remains unclear and may be related to processing of contextual information, rather than object representation per se (Gaffan, 1994; Cassaday and Rawlins, 1997; Bussey and Aggleton, 2002; Mumby et al., 2002; Aggleton and Brown, 2005; O’Brien et al., 2006; Piterkin et al., 2008).

Evidence now suggests that the reconsolidation process is involved in modification of established memories (Hupbach et al., 2007; Lukowiak et al., 2007; Lee, 2008, 2010; Inda et al., 2011). Accordingly, new learning, or the presence of novel stimuli, at the time of memory reactivation can influence the likelihood of memories to undergo reconsolidation (Rodriguez-Ortiz et al., 2005, 2008; Morris et al., 2006; Winters et al., 2009). Moreover, previous findings have suggested a role for the HPC in object memory reconsolidation only when memory updating occurs (Rossato et al., 2007). In the Rossato et al. (2007) study, hippocampal protein synthesis inhibition impaired object memory reconsolidation only when reactivation occurred in the presence of a novel object. This study, however, used an open field version of the SOR task, and there are inconsistencies in the literature regarding hippocampal involvement depending on the procedure used to assess object recognition (Winters et al., 2008). One aim of the current study was therefore to investigate the nature of 
novel information required at memory reactivation to prompt involvement of the HPC in reconsolidation of object memories. Given its importance in object information processing, it is also surprising that no studies have assessed the role of PRh in object memory reconsolidation. In the current study we therefore conducted a systematic assessment of the contributions of the PRh and HPC to object memory reconsolidation using variants of the SOR task in which novel object or contextual information was presented during reactivation of the original object memory.

\section{Materials and Methods}

Subjects. The subjects were 117 adult male Long-Evans rats (Charles River), weighing $\sim 300-350 \mathrm{~g}$ at the onset of each experiment. Rats were housed in pairs and maintained on a reverse light/dark cycle (7:00 A.M. lights off; 7:00 P.M. lights on). All behavioral testing occurred during the dark phase of the cycle, and the experimental room was illuminated by overhead lighting during testing. Rats received $20 \mathrm{~g}$ of rodent chow each evening to maintain $85-90 \%$ of free-feeding body weight for the duration of the behavioral phase of each experiment. Water was available ad libitum throughout the experiments. All procedures adhered to the guidelines of the Canadian Council on Animal Care and were approved by the Animal Care Committee at the University of Guelph.

Surgical procedures. For each experiment, all rats were implanted bilaterally with 22-gauge indwelling guide cannulas according to the following procedure. Before and during all surgeries, rats were deeply anesthetized with isoflurane inhalation anesthetic (Benson Medical Industries). Animals also received a systemic (subcutaneous) injection of the analgesic meloxicam ( $5 \mathrm{mg} / \mathrm{ml}$; Boehringer Ingelheim) before surgery. They were then placed in a stereotaxic frame (Kopf Instruments) with the incisor bar set to $-3.3 \mathrm{~mm}$. The scalp was cut and retracted to expose the skull, and holes were drilled directly above the target region. The guide cannulas were implanted according to the following coordinates, measured relative to the skull at bregma (Paxinos and Watson, 1998), for PRh: anteroposterior $-5.5 \mathrm{~mm}$, lateral $\pm 6.6 \mathrm{~mm}$, dorsoventral $-6.5 \mathrm{~mm}$; for HPC: anteroposterior $-3.8 \mathrm{~mm}$, lateral $\pm 2.5 \mathrm{~mm}$, dorsoventral $-2.5 \mathrm{~mm}$. The cannulas were secured to the skull using four jeweler screws and dental acrylic. Dummy cannulas cut to extend $1.1 \mathrm{~mm}$ beyond the tip of the guide cannulas and with an outer diameter of 0.36 $\mathrm{mm}$ were inserted into the guides and remained there except during infusions. At the completion of surgery, the skin was sutured, and animals recovered in cages on heat pads for $1-2 \mathrm{~h}$ before being returned to their home cages. Rats were allowed to recover in their home cages for at least $7 \mathrm{~d}$ before the beginning of behavioral testing.

Drugs. The protein synthesis inhibitor anisomycin (Sigma) was initially dissolved in $1 \mathrm{~N} \mathrm{HCl}$. The $\mathrm{pH}$ of the solution was then adjusted to 7.0 by addition of $\mathrm{NaOH}$, and the final concentration was adjusted to 100 $\mu \mathrm{g} / \mu \mathrm{l}$ by adding $0.9 \%$ physiological saline. This dose was chosen because previous studies have indicated that anisomycin in a dose rang of $100-$ $125 \mu \mathrm{g} / \mu \mathrm{l}$ produces $>90 \%$ protein synthesis inhibition in various brain regions (Rosenblum et al., 1993; Morris et al., 2006).

Infusion procedure. For all experiments, rats received bilateral infusions of either $0.9 \%$ physiological saline or anisomycin on a given trial, immediately following the reactivation phase (or at an equivalent time within the trial for "no reactivation" conditions, see below). All experiments were run with drug as a within-subjects factor, and rats received counterbalanced infusions of anisomycin or saline over multiple trials (see below). All infusions took place in a preparation room separate from the behavioral testing area. Animals were gently restrained by the experimenter throughout the infusion process. The dummy cannulas were removed, and the 28-gauge infusion cannulas, which were cut to extend $1 \mathrm{~mm}$ beyond the tip of the guide cannulas, were inserted. Bilateral infusions were conducted simultaneously using two $1 \mu$ l Hamilton syringes, which were connected to the infusion cannulas by propylene tubing. The syringes were driven by a Harvard Apparatus precision syringe pump, which delivered $1 \mu \mathrm{l}$ to each hemisphere over $2 \mathrm{~min}$. Previous results demonstrate that various infusates spread within the brain with a radius of $\sim 1-2 \mathrm{~mm}$ from the site of infusion when administered in this volume (Martin, 1991; Berman et al., 2000), thereby limiting diffu- sion into adjacent untargeted brain regions. This is consistent with our own previous $\mathrm{PRh}$ analyses showing that the infusion parameters used in the current study produce drug diffusion patterns predominantly limited to PRh, with very little, if any, encroachment into adjacent hippocampal or cortical structures, including lateral entorhinal cortex (Winters and Bussey, 2005b; Winters et al., 2010). The infusion cannulas were left in place for an addition $1.5 \mathrm{~min}$ to allow for diffusion of the infusate. The infusion cannulas were then removed, and the dummy cannulas reinserted. Rats were then returned to their home cages until the choice phase. For all experiments, in each of the two habituation sessions before the beginning of behavioral testing, rats experienced a mock infusion identical to the procedure described above, except that the injection cannulas contained no liquid.

Apparatus. Spontaneous object recognition was conducted in a Y-shaped apparatus as described previously (Winters et al., 2004; Forwood et al., 2005). Briefly, the Y-shaped apparatus had high, homogenous white walls constructed from Plexiglas to prevent the rat from looking out into the room. The apparatus walls were $40 \mathrm{~cm}$ high, and each arm was $27 \mathrm{~cm}$ in length and $10 \mathrm{~cm}$ wide. The start arm contained a guillotine door $18 \mathrm{~cm}$ from the rear of the arm. This provided an area within which the rat could be confined at the start of a given trial. A video camera was mounted on a tripod above the apparatus to record all trials. Duplicate copies of objects made from plastic, ceramic, glass, and aluminum were obtained. The height of the objects ranged from 10 to $20 \mathrm{~cm}$, and they varied with respect to their visual and tactile qualities. All objects were affixed to the floor of the apparatus with an odorless reusable adhesive putty to prevent them from being displaced during testing. As far as could be determined, the objects had no natural significance for the rats, and they had never been associated with a reinforcer. Before being placed in the apparatus, objects were always wiped with $50 \%$ ethanol.

Spontaneous object recognition. Each of the seven experiments followed the same general procedure. Specific experimental manipulations are outlined below. All rats were habituated to the empty Y-shaped apparatus in two consecutive daily sessions before the start of trials. Each rat was individually brought to the experiment room in a transport cage, placed in the start box area of the Y-shaped apparatus, and released into the main exploration area for $5 \mathrm{~min}$. Immediately following the habituation session, each rat received a mock infusion as outlined above.

Behavioral testing began $24 \mathrm{~h}$ after the second habituation session. All rats were run for four trials total in each experiment, two trials with saline administration and two with anisomycin, with at least $48 \mathrm{~h}$ between trials. Each trial consisted of three phases: sample, reactivation, and choice. Each phase occurred $24 \mathrm{~h}$ apart, for a retention delay (sample-tochoice) of $48 \mathrm{~h}$ on each trial. A different object pair was used for each trial for a given rat, and the order of exposure to object pairs as well as the designated sample and novel objects for each pair were counterbalanced. Time spent exploring the objects was assessed using video recordings of the sample, reactivation, and choice phases. Data were collected by scoring exploratory bouts using a personal computer.

In the sample phase, two identical objects were placed in the Y-shaped apparatus, one at the end of each exploration arm. Each rat was individually brought into the experiment room in a transport box and placed in the start box with the guillotine door lowered. The guillotine door was then raised to allow the rat into the exploration area of the Y-shaped apparatus. When the rat exited the start box, the guillotine door was lowered to prevent reentry, and the sample phase began. The time spent exploring the two objects was scored by an experimenter viewing the rat on a video screen. Exploration of an object was defined as directing the nose to the object at a distance of $<2 \mathrm{~cm}$ and/or touching it with the nose. The sample phase ended when the rat had explored the identical objects for a total of $25 \mathrm{~s}$, or after $3 \mathrm{~min}$ had passed, whichever came first. At the end of the sample phase, the rat was removed from the Y-shaped apparatus and was transported back to its home cage.

We modified the SOR paradigm to assess object memory reconsolidation with three different memory reactivation conditions interposed between the sample and choice phases: "standard," "novel object," and "context change" reactivation conditions. For the reactivation phase in each experiment, each rat was individually brought to the experiment room in a transport cage. For the standard reactivation condition (Ex- 
periments 1 and 2), the Y-shaped apparatus contained the same objects shown to the rat during the sample phase; the reactivation phase was performed identically to the sample phase, except that it ended when the rat had explored the objects for a total of $10 \mathrm{~s}$, or when $2 \mathrm{~min}$ had passed, whichever came first. The novel object reactivation condition (Experiments 3 and 4 ) was run in the same manner as the standard reactivation, except that one of the sample objects was replaced by a new object in the reactivation phase; the side of the novel object was counterbalanced across rats, and the novel object presented in the reactivation phase was different from the novel object presented in the choice phase. The context change reactivation condition (Experiments 5-7) was identical to the standard reactivation, except that a textural insert constructed of white foam board and covered in no-slip rubber padding was placed on the floor of the main stem of the Y-shaped apparatus for the reactivation phase. The insert was $10 \mathrm{~cm}$ wide, and extended $38 \mathrm{~cm}$ from inside the start box into the exploration area; the floor insert did not extend into the arms containing the objects, but covered the entire "middle stem" of the Y-shaped apparatus, extending beyond the start box into the exploratory area. This textural insert was used in a previous study and found to be effective in destabilizing certain object memories when presented at reactivation (Winters et al., 2009). For ease of insertion into the Y-shaped apparatus and to maintain the stability of the objects being presented, the insert did not extend into the presentation arms of the Y-shaped apparatus. Following the reactivation phase, the rat was removed and immediately administered an intracranial infusion of anisomycin or saline as described above. For animals in the no reactivation condition, the reactivation phase was omitted, and rats were administered infusions in the same manner and at the same time following the sample phase as animals in the reactivation groups. The experimenter was blind to the substance administered. Each rat was run on two trials with each drug condition, and the order of drug administration was counterbalanced within and between experimental groups. Each rat was run for four trials total in each experiment because this produces data with less variability in the SOR task. Following the infusion, the rat was returned to its home cage until the start of the choice phase.

In the choice phase, which occurred $24 \mathrm{~h}$ after the reactivation phase in all experiments, the Y-shaped apparatus contained a copy of the original sample object in one arm and a new object in the other. The exploration arms in which the novel and sample objects were placed for the choice phase were counterbalanced across trials. The rat was allowed to explore the objects for $2 \mathrm{~min}$. The time spent exploring the novel and familiar objects was recorded for the $2 \mathrm{~min}$ of the choice phase. We calculated a discrimination ratio, i.e., the difference in time spent exploring the novel and familiar objects divided by the total time spent exploring the objects, for the first minute of the choice phase on each object recognition trial [i.e., (novel choice object exploration - familiar choice object exploration)/total choice object exploration]. The first minute was assessed because we have consistently observed that this is when the maximal discrimination is shown between novel and familiar objects by normal animals (our unpublished observations; see also Dix and Aggleton, 1999). Normal rats tend to explore the novel object more than the familiar sample object in this spontaneous object recognition paradigm.

Experiment 1. Experiment 1 assessed the role of PRh in object memory reconsolidation using the standard reactivation condition. Two groups of rats were tested. Rats in the "reactivation" group $(n=8)$ received intra-PRh infusions of anisomycin or saline immediately following a standard reactivation phase, as described above. Rats in the no reactivation group $(n=8)$ received infusions of anisomycin or saline at approximately the same time within trials ( $24 \mathrm{~h}$ after the sample phase), but did not undergo a reactivation phase.

Experiment 2. Experiment 2 investigated the involvement of the HPC in object memory reconsolidation using the standard reactivation condition. Rats in the reactivation group $(n=9)$ received intra-HPC infusions of anisomycin or saline immediately following a standard reactivation phase, as described above. Rats in the no reactivation group $(n=8)$ were treated as described for Experiment 1.

Experiment 3. Experiment 3 assessed the involvement of PRh in object memory reconsolidation with a novel object presented at the time of memory reactivation. All procedures were identical to those in Exper- iment 1 , except that the reactivation group $(n=10)$ experienced the novel object reactivation phase, as described above, before drug infusions. Rats in the no reactivation group $(n=9)$ were treated as described for Experiment 1.

Experiment 4. Experiment 4 tested the involvement of the HPC in object memory reconsolidation with a novel object presented at the time of memory reactivation. All procedures were identical to those in Experiment 2, except that the reactivation group $(n=8)$ experienced the novel object reactivation phase, as described above, before drug infusions. Rats in the no reactivation group $(n=8)$ were treated as described for Experiment 1.

Experiment 5. Experiment 5 investigated the role of PRh in object memory reconsolidation with a novel contextual cue presented at the time of memory reactivation. All procedures were identical to those in Experiment 1, except that the reactivation group $(n=6)$ experienced the context change reactivation phase, as described above, before drug infusions. Rats in the no reactivation group $(n=8)$ were treated as described for Experiment 1.

Experiment 6. Experiment 6 assessed the role of the HPC in object memory reconsolidation with a novel contextual cue presented at the time of memory reactivation. All procedures were identical to those in Experiment 2, except that the reactivation group $(n=7)$ experienced the context change reactivation phase, as described above, before drug infusions. Rats in the no reactivation group $(n=9)$ were treated as described for Experiment 1.

Experiment 7. Experiment 7 explored the functional interaction between the HPC and PRh in object memory reconsolidation with a novel contextual cue presented at the time of memory reactivation. All rats were implanted bilaterally with cannulas in both PRh and dorsal HPC. All procedures were identical to those in Experiment 5 except that the reactivation group $(n=10)$ received crossed unilateral infusions of anisomycin or saline into PRh and HPC immediately following the context change reactivation phase. For instance, a rat receiving an intra-PRh infusion into the left hemisphere was simultaneously administered an intra-HPC infusion into the right hemisphere, or vice versa. The side of injection for each brain region was counterbalanced across all subjects and trials. Rats in the no reactivation group $(n=9)$ received the same crossed unilateral injection procedure but did not experience the reactivation phase.

Histology. Following behavioral testing, rats were anesthetized by intraperitoneal injection of $2 \mathrm{ml}$ of Euthansol ( $340 \mathrm{mg} / \mathrm{ml}$; Schering Canada Inc.) and perfused transcardially with $100 \mathrm{ml}$ of PBS, pH 7.4, followed by $250 \mathrm{ml}$ of $4 \%$ neutral buffered formalin, $\mathrm{pH} 7.4$ (EMD). The brains were removed, postfixed in $4 \%$ formalin at $4^{\circ} \mathrm{C}$ for at least $24 \mathrm{~h}$ and then immersed in $20 \%$ sucrose in PBS until they sank. A cryostat was used to cut coronal sections $(60 \mu \mathrm{m})$ through the extent of PRh or HPC, and every fifth section was mounted on a gelatin-coated glass slide and stained with cresyl violet. Slides were examined under a light microscope to verify the cannula placements.

Data analysis. Group means of six measures taken from object recognition testing were analyzed for each experiment. Two measures were analyzed from each phase of behavioral testing: the total object exploration in the sample phase and the duration of the sample phase; the total object exploration and duration of the reactivation phase; the total object exploration and the discrimination ratio from the choice phase. Means for the four measures from the sample and choice phases were submitted to two-way (drug $\times$ reactivation group) mixed-factors analyses of variance (ANOVA), with paired-samples $t$ tests used for post hoc analyses in cases of significant interactions. Means for the two measures taken from the reactivation phase were compared across drug conditions using paired-samples $t$ tests. An additional analysis was performed on the object recognition data for each experiment using independent samples $t$ tests to compare the mean discrimination ratio in each condition to a score of 0 (i.e., "chance" performance, no significant discrimination between the novel and familiar objects in the choice phase). All statistical analyses were conducted with a significance level of $\alpha=0.05$ using SPSS 18.0 for Windows. 

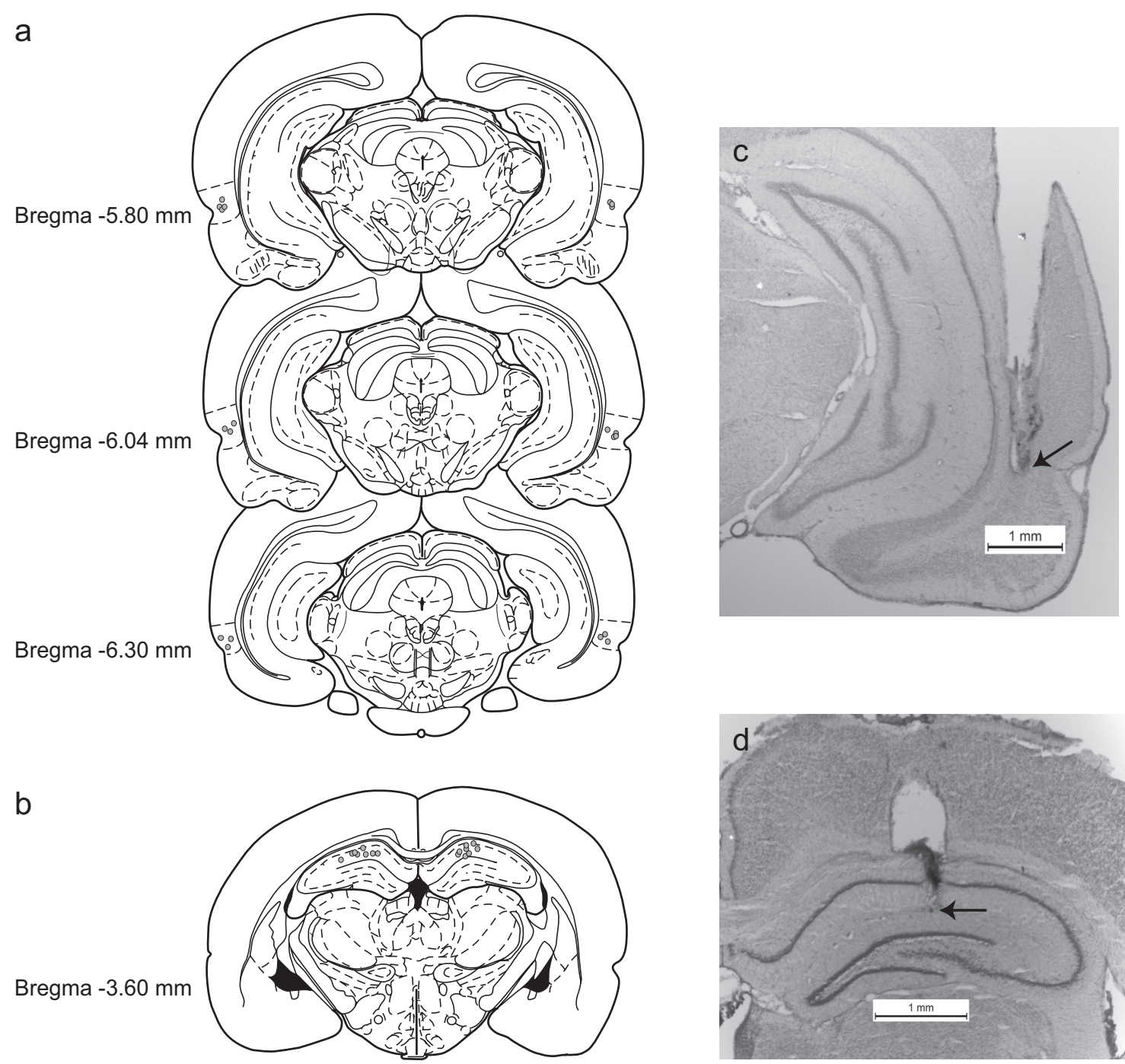

Figure 1. $\quad \boldsymbol{a}, \boldsymbol{b}$, Schematic representation of the infusion needle tip placements from typical groups of animals with PRh implantations (a) (Experiment 3 , reactivation group; $n=10)$ and HPC implantations $(\boldsymbol{b})$ (Experiment 4, reactivation group; $n=8$ ). These placements are representative of needle tip locations in all animals included in the behavioral analyses of the present study. Cannulas in PRh were consistently located between 5.80 and $6.30 \mathrm{~mm}$ posterior to bregma. Cannulas in HPC for all animals were located at $\sim 3.6 \mathrm{~mm}$ posterior to bregma. Some needle tips overlap in the figure. Brain section illustrations modified from Paxinos and Watson (1998). c and $\boldsymbol{d}$ are photomicrographs illustrating PRh and dorsal HPC cannula tracks, respectively; arrows indicate tips of infusion cannula tracks. Dashed lines surrounding placements in $\boldsymbol{a}$ approximate the borders of PRh as defined by Burwell (2001).

\section{Results}

\section{Histology}

All rats included in the behavioral analyses for Experiments 1, 3, and 5 had guide cannulas located bilaterally with injection needle tips terminating in PRh near the border between areas 35 and 36 (Burwell, 2001); these placements were consistently located between 5.80 and $6.30 \mathrm{~mm}$ posterior to bregma (Fig. 1a,c). All rats included in the behavioral analyses for Experiments 2, 4, and 6 had guide cannulas located bilaterally with injection needle tips terminating in the dorsal HPC (Fig. 1b,d). For Experiment 7, all rats had bilateral placements of cannulas in both dorsal HPC and PRh.

\section{Experiment 1: Protein synthesis in PRh is required for reconsolidation of object memories following standard reactivation}

Rats receiving bilateral infusions of anisomycin into PRh immediately following the reactivation phase failed to show a preference for the novel object in the choice phase (Fig. 2). Mixed-factors ANOVA revealed nonsignificant drug $\left(F_{(1,14)}=3.74, p=0.074\right)$ and group $\left(F_{(1,14)}=1.74, p=0.208\right)$ effects, but a significant drug $\times$ group interaction $\left(F_{(1,14)}=5.29, p=0.037\right)$. Post hoc analysis with paired-samples $t$ tests indicated a significant difference between saline and anisomycin conditions within the reactivation group $\left(t_{(7)}=2.95, p=0.021\right)$, but not within the no reactivation group $\left(t_{(7)}=0.264, p=0.799\right)$. This pattern of effects was consistent with the results of separate independent-samples $t$ tests, which indicated that rats showed significant novel object preference (i.e., discrimination ratios significantly $>0$ ) in all conditions (reactivation-saline, $t_{(7)}=3.963, p=0.005$; no reactivation-saline, $t_{(7)}=2.303, p=0.05$; no reactivation-anisomycin, $t_{(7)}=6.002, p=$ 0.001 ), except when receiving anisomycin into PRh immediately after the reactivation phase $\left(t_{(7)}=0.382, p=0.714\right)$.

Analysis of the general exploration measures indicated no significant differences between conditions in terms of total exploration levels (sample: drug, $F_{(1,14)}=0.113, p=0.742$, group, $F_{(1,14)}=0.33, p=0.575$, drug $\times$ group, $F_{(1,14)}=1.06, p=0.322$; reactivation: $t_{(7)}=1.19, p=0.273$; choice: $\operatorname{drug}, F_{(1,14)}=0.496, p=$ 0.493 , group, $F_{(1,14)}=0.045, p=0.835$, drug $\times$ group, $F_{(1,14)}=$ $0.816, p=0.382$ ) or phase duration (sample: drug, $F_{(1,14)}=4.21$, 


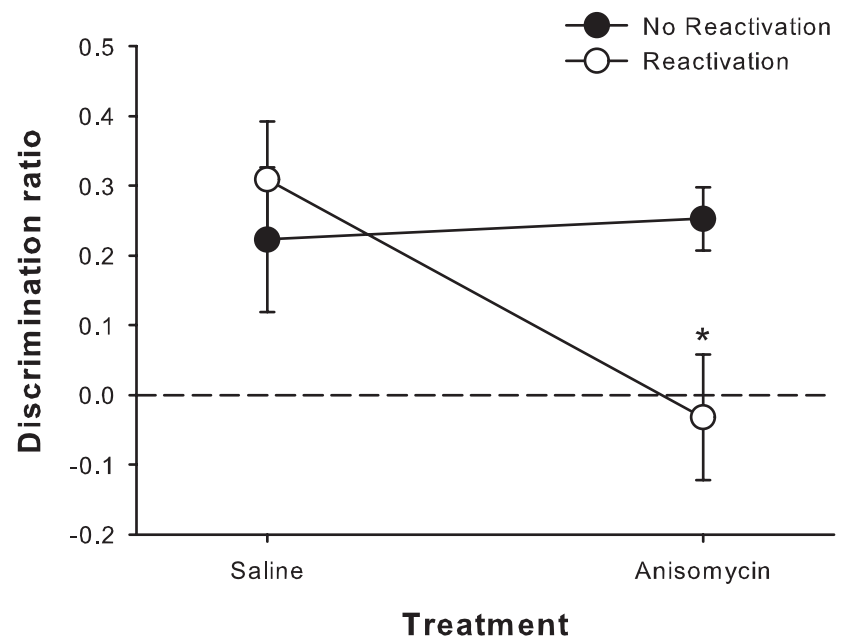

Figure 2. Spontaneous object recognition performance of rats in Experiment 1. Intra-PRh administration of anisomycin immediately following the standard reactivation phase prevented object memory reconsolidation. Perirhinal protein synthesis inhibition did not affect object recognition memory in rats that did not undergo a memory reactivation phase before drug delivery. Data are presented as average discrimination ratio ( \pm SEM) from the choice phase administered $24 \mathrm{~h}$ after drug infusions. ${ }^{*} p<0.05$ (anisomycin vs saline in reactivation group).

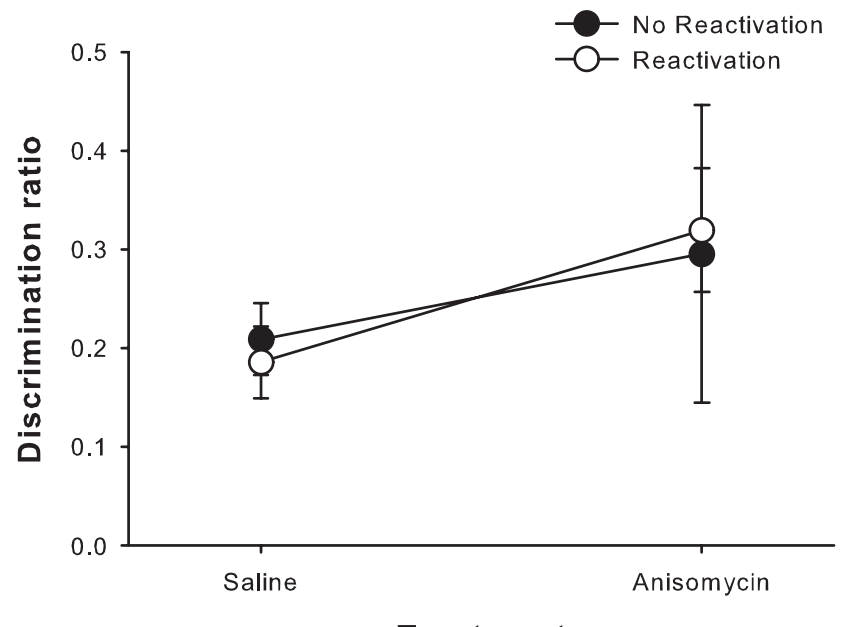

Treatment

Figure 3. Spontaneous object recognition performance of rats in Experiment 2. Intra-HPC administration of anisomycin immediately following the standard reactivation phase did not affect object memory reconsolidation. Data are presented as average discrimination ratio ( \pm SEM) from the choice phase administered $24 \mathrm{~h}$ after drug infusions.

$p=0.059$, group, $F_{(1,14)}=0.913, p=0.355$, drug $\times$ group, $F_{(1,14)}=$ $1.996, p=0.18$; reactivation: $\left.t_{(7)}=0.255, p=0.806\right)$.

Experiment 2: Protein synthesis in dorsal HPC is not required for reconsolidation of object memories following standard reactivation

Bilateral infusions of anisomycin into the HPC immediately following the reactivation phase did not disrupt preference for the novel object in the choice phase (Fig. 3). Mixed-factors ANOVA revealed nonsignificant drug $\left(F_{(1,15)}=2.074, p=0.17\right)$, group $\left(F_{(1,15)}=0.0001, p=0.995\right)$, and drug $\times$ group interaction $\left(F_{(1,15)}=0.095, p=0.762\right)$ terms. This pattern of effects was consistent with the results of separate independent-samples $t$ tests, which indicated that rats showed significant novel object preference in all conditions (reactivation-saline, $t_{(8)}=5.387$,

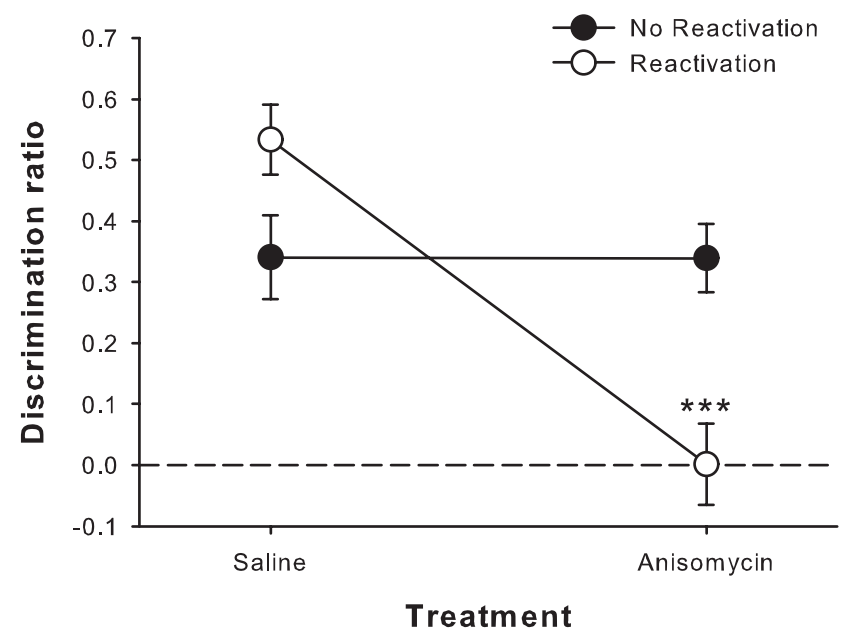

Figure 4. Spontaneous object recognition performance of rats in Experiment 3. Intra-PRh administration of anisomycin immediately following memory reactivation in the presence of a novel object prevented reconsolidation of the sample object trace. Inhibition of protein synthesis in PRh did not affect object recognition memory in rats that did not undergo a memory reactivation phase before drug delivery. Data are presented as average discrimination ratio ( \pm SEM) from the choice phase administered $24 \mathrm{~h}$ after drug infusions. ${ }^{* * *} p<0.001$ (anisomycin vs saline in reactivation group).

$p=0.001 ;$ reactivation-anisomycin, $t_{(8)}=5.41, p=0.001 ;$ no reactivation-anisomycin, $t_{(7)}=2.094, p=0.037$, one-tailed; no reactivation-saline, $t_{(7)}=6.147, p<0.001$ ).

There were also no significant differences between conditions in terms of total exploration levels (sample: drug, $F_{(1,15)}=1.44, p=$ 0.248 , group, $F_{(1,15)}=0.794, p=0.387$, drug $\times$ group, $F_{(1,15)}=$ $0.407, p=0.533$; reactivation: $t_{(8)}=0.294, p=0.776$; choice: drug, $F_{(1,15)}=2.766, p=0.117$, group, $F_{(1,15)}=0.118, p=0.735$, drug $\times$ group, $F_{(1,15)}=0.071, p=0.794$ ) or phase duration (sample: drug, $F_{(1,15)}=3.918, p=0.066$, group, $F_{(1,15)}=0.25, p=0.624$, drug $\times$ group, $F_{(1,15)}=0.059, p=0.811$; reactivation: $\left.t_{(8)}=0.69, p=0.51\right)$.

Experiment 3: Protein synthesis in PRh is required for reconsolidation of object memories following reactivation in the presence of a novel object

Bilateral infusions of anisomycin into PRh immediately following memory reactivation in the presence of a novel object significantly impaired object recognition in the choice phase (Fig. 4). Mixed-factors ANOVA revealed a nonsignificant effect of group $\left(F_{(1,17)}=1.398, p=0.253\right)$, but a significant drug effect $\left(F_{(1,17)}=\right.$ $17.83, p=0.001)$ and a significant drug $\times$ group interaction $\left(F_{(1,17)}=17.639, p=0.001\right)$. Post hoc analysis with pairedsamples $t$ tests indicated a significant difference between saline and anisomycin conditions within the reactivation group $\left(t_{(9)}=\right.$ $6.186, p<0.001)$, but not within the no reactivation group $\left(t_{(8)}=\right.$ $0.015, p=0.988)$. This pattern of effects was supported by the results of separate independent-samples $t$ tests, which indicated significant novel object preference in all conditions (reactivationsaline, $t_{(9)}=9.378, p<0.001$; no reactivation-saline, $t_{(8)}=4.991$, $p=0.001$; no reactivation-anisomycin, $\left.t_{(8)}=6.005, p<0.001\right)$, except when rats received anisomycin into PRh immediately after the reactivation phase $\left(t_{(9)}=0.016, p=0.988\right)$.

General exploration analyses indicated no significant differences between conditions in terms of total exploration levels (sample: drug, $F_{(1,17)}=0.09, p=0.768$, group, $F_{(1,17)}=0.354$, $p=0.56$, drug $\times$ group, $F_{(1,17)}=0.22, p=0.645$; reactivation: $t_{(9)}=1.329, p=0.216$; choice: drug, $F_{(1,17)}=0.129, p=0.724$, group, $F_{(1,17)}=1.89, p=0.187$, drug $\times$ group, $F_{(1,17)}=1.282$, 


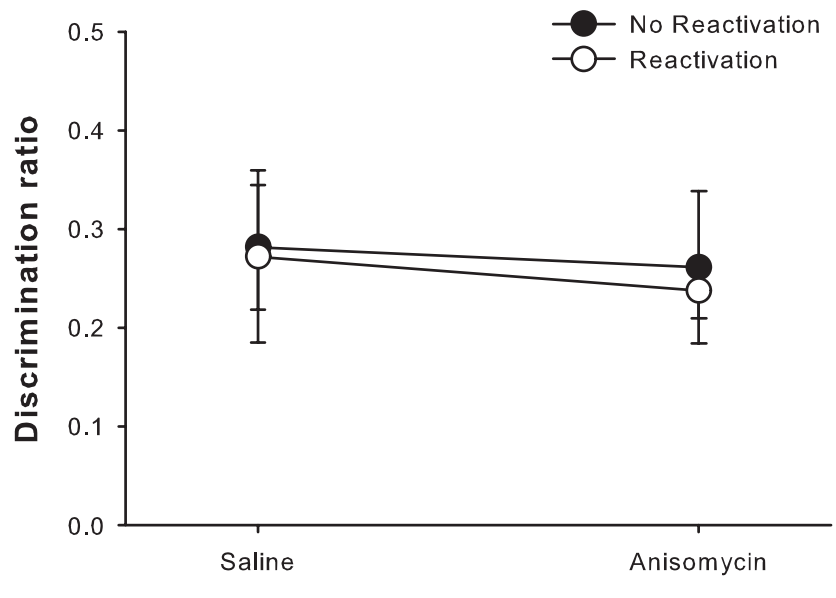

Treatment

Figure 5. Spontaneous object recognition performance of rats in Experiment 4. Intra-HPC administration of anisomycin immediately following memory reactivation in the presence of a novel object did not affect reconsolidation of the sample object trace. Data are presented as average discrimination ratio ( \pm SEM) from the choice phase administered $24 \mathrm{~h}$ after drug infusions.

$p=0.273$ ) or phase duration (sample: drug, $F_{(1,17)}=0.089, p=$ 0.769 , group, $F_{(1,17)}=2.697, p=0.119$, drug $\times$ group, $F_{(1,17)}=$ $0.004, p=0.948$; reactivation: $\left.t_{(9)}=1.882, p=0.093\right)$.

Experiment 4: Protein synthesis in dorsal HPC is not required for reconsolidation of object memories following reactivation in the presence of a novel object

Rats receiving bilateral infusions of anisomycin into the HPC immediately following memory reactivation in the presence of a novel object displayed intact object recognition memory in the choice phase (Fig. 5). Mixed-factors ANOVA revealed nonsignificant drug $\left(F_{(1,14)}=0.226, p=0.642\right)$, group $\left(F_{(1,14)}=0.047, p=\right.$ $0.832)$, and drug $\times$ group interaction $\left(F_{(1,14)}=0.015, p=0.905\right)$ terms. Accordingly, separate independent-samples $t$ tests indicated that rats showed significant novel object preference in all conditions (reactivation-saline, $t_{(7)}=3.131, p=0.017$; reactivation-anisomycin, $t_{(7)}=8.535, p<0.001$; no reactivationsaline, $t_{(7)}=4.453, p<0.003$; no reactivation-anisomycin, $t_{(7)}=$ 3.385, $p=0.012$ ).

There were also no significant differences between conditions in terms of total exploration levels (sample: drug, $F_{(1,14)}=0.329, p=$ 0.575 , group, $F_{(1,14)}=0.318, p=0.582$, drug $\times$ group, $F_{(1,14)}=$ 1.112, $p=0.31$; reactivation: $t_{(7)}=1.347, p=0.22$; choice: drug, $F_{(1,14)}=0.199, p=0.663$, group, $F_{(1,14)}=0.003, p=0.954$, drug $\times$ group, $F_{(1,14)}=0.004, p=0.953$ ) or phase duration (sample: drug, $F_{(1,14)}=0.343, p=0.568$, group, $F_{(1,14)}=0.008, p=0.93$, drug $\times$ group, $F_{(1,14)}=1.07, p=0.319$; reactivation: $t_{(7)}=$ $0.394, p=0.705)$.

\section{Experiment 5: Protein synthesis in PRh is required for reconsolidation of object memories following reactivation in an altered context}

Bilateral infusions of anisomycin into PRh immediately following memory reactivation in the presence of a novel contextual cue significantly disrupted object recognition in the choice phase (Fig. 6). Mixed-factors ANOVA revealed a nonsignificant effect of group $\left(F_{(1,12)}=0.649, p=0.436\right)$, but trends toward significant drug $\left(F_{(1,12)}=3.598, p=0.082\right)$ and drug $\times$ group interaction $\left(F_{(1,12)}=3.271, p=0.096\right)$ effects. Moreover, planned comparisons with paired-samples $t$ tests indicated a significant

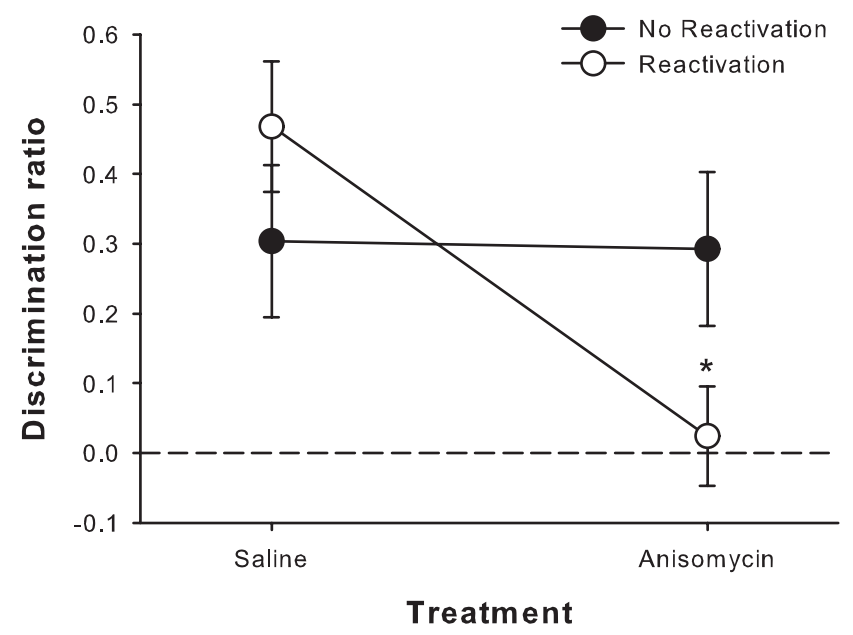

Figure 6. Spontaneous object recognition performance of rats in Experiment 5. Intra-PRh administration of anisomycin immediately following memory reactivation in the presence of a novel contextual cue blocked reconsolidation of the sample object trace. Perirhinal protein synthesis inhibition did not affect object recognition memory in rats that did not undergo a memory reactivation phase before drug delivery. Data are presented as average discrimination ratio ( \pm SEM) from the choice phase administered $24 \mathrm{~h}$ after drug infusions. ${ }^{*} p<0.05$ (anisomycin vs saline in reactivation group).

difference between saline and anisomycin conditions within the reactivation group $\left(t_{(5)}=2.997, p=0.03\right)$, but not within the no reactivation group $\left(t_{(7)}=0.061, p=0.953\right)$. Furthermore, the interpretation of object recognition impairment was supported by the results of separate independent-samples $t$ tests, which indicated significant novel object preference in all conditions (reactivation-saline, $t_{(5)}=5.508, p=0.003$; no reactivationsaline, $t_{(7)}=2.975, p=0.021$; no reactivation-anisomycin, $t_{(7)}=2.826, p<0.026$ ), except when rats received intra-PRh anisomycin immediately after the reactivation phase $\left(t_{(5)}=\right.$ $0.368, p=0.728)$.

Analysis of general exploration values indicated no significant differences between conditions in terms of total exploration levels (sample: drug, $F_{(1,12)}=3.134, p=0.102$, group, $F_{(1,12)}=0.215, p=$ 0.651 , drug $\times$ group, $F_{(1,12)}=0.496, p=0.495$; reactivation: $t_{(5)}=$ $0.282, p=0.79$; choice: drug, $F_{(1,12)}=0.347, p=0.568$, group, $F_{(1,12)}=0.073, p=0.792$, drug $\times$ group, $\left.F_{(1,12)}=0.01, p=0.923\right)$ or phase duration (sample: drug, $F_{(1,12)}=0.177, p=0.681$, group, $F_{(1,12)}=0.022, p=0.886$, drug $\times$ group, $F_{(1,12)}=0.278, p=0.608$; reactivation: $\left.t_{(5)}=1.581, p=0.175\right)$.

Experiment 6: Protein synthesis in dorsal HPC is required for reconsolidation of object memories following reactivation in an altered context

Unlike in the standard and novel object reactivation conditions, bilateral infusions of anisomycin into the HPC immediately following memory reactivation in the presence of a novel contextual cue significantly impaired object recognition in the choice phase (Fig. 7). Mixed-factors ANOVA revealed a nearly significant effect of $\operatorname{group}\left(F_{(1,14)}=4.471, p=0.053\right)$, as well as a significant drug effect $\left(F_{(1,14)}=5.899, p=0.029\right)$ and a significant drug $\times$ group interaction $\left(F_{(1,14)}=8.6, p=0.011\right)$. Post hoc analysis with paired-samples $t$ tests indicated a significant difference between saline and anisomycin conditions within the reactivation group $\left(t_{(6)}=3.518, p<0.013\right)$, but not within the no reactivation group $\left(t_{(8)}=0.386, p=0.71\right)$. This pattern of effects was reflected in the results of separate independent-samples $t$ tests, which indicated significant novel object preference in all conditions (reactivation- 


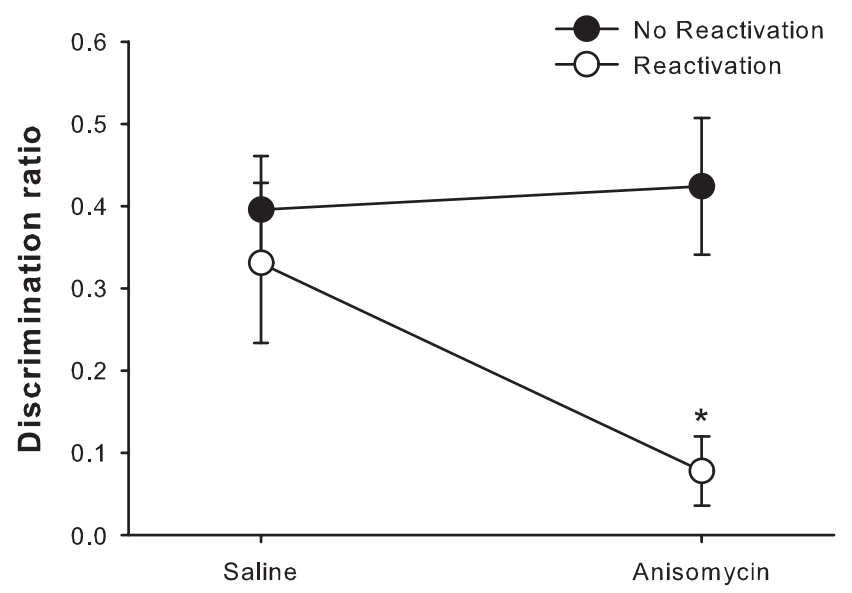

Treatment

Figure 7. Spontaneous object recognition performance of rats in Experiment 6. Intra-HPC administration of anisomycin immediately following memory reactivation in the presence of a novel contextual cue prevented reconsolidation of the sample object trace. Inhibition of hippocampal protein synthesis did not affect object recognition memory in rats that did not undergo a memory reactivation phase before drug delivery. Data are presented as average discrimination ratio ( \pm SEM) from the choice phase administered $24 \mathrm{~h}$ after drug infusions. ${ }^{*} p<0.05$ (anisomycin vs saline in reactivation group).

saline, $t_{(6)}=4.249, p<0.005$; no reactivation-saline, $t_{(8)}=6.437$, $p<0.001$; no reactivation-anisomycin, $t_{(8)}=5.398, p=0.001$ ), except when rats received anisomycin into the HPC immediately after the reactivation phase $\left(t_{(6)}=1.771, p=0.127\right)$.

General exploration analyses indicated no significant differences for total exploration levels (sample: drug, $F_{(1,14)}=0.324, p=0.578$, group, $F_{(1,14)}=0.245, p=0.628$, drug $\times$ group, $F_{(1,14)}=1.383, p=$ 0.259 ; reactivation: $t_{(6)}=0.888, p=0.409$; choice: drug, $F_{(1,14)}=$ $0.541, p=0.474$, group, $F_{(1,14)}=2.509, p=0.136$, drug $\times$ group, $F_{(1,14)}=0.313, p=0.585$ ) or phase duration (sample: drug, $F_{(1,14)}=$ $0.038, p=0.848$, group, $F_{(1,14)}=0.201, p=0.661$, drug $\times$ group, $F_{(1,14)}=0.028, p=0.87$; reactivation: $\left.t_{(6)}=0.368, p=0.726\right)$.

\section{Experiment 7: Coordinated protein synthesis in PRh and $\mathrm{HPC}$ is required for reconsolidation of object memories following reactivation in an altered context}

Having demonstrated equivalent involvement of PRh and HPC in Experiments 5 and 6, we next investigated the possibility that these two structures functionally interact to mediate object memory reconsolidation following reactivation in the presence of a salient novel contextual cue. Crossed unilateral infusions of anisomycin into PRh and HPC immediately following memory reactivation in the context change condition significantly disrupted object recognition in the choice phase (Fig. 8). Mixed-factors ANOVA revealed a nonsignificant effect of group $\left(F_{(1,17)}=\right.$ $0.186, p=0.672)$, but a significant drug effect $\left(F_{(1,17)}=10.87\right.$, $p=0.004)$ and a significant drug $\times$ group interaction $\left(F_{(1,17)}=\right.$ 8.304, $p=0.01)$. Post hoc analysis with paired-samples $t$ tests indicated a significant difference between saline and anisomycin conditions within the reactivation group $\left(t_{(9)}=3.729, p=\right.$ $0.005)$, but not within the no reactivation group $\left(t_{(8)}=0.406, p=\right.$ 0.695). This pattern of effects was supported by the results of separate independent-samples $t$ tests, which indicated significant novel object preference in all conditions (reactivation-saline, $t_{(9)}=$ 7.467, $p<0.001$; no reactivation-saline, $t_{(8)}=5.14, p=0.001$; no reactivation-anisomycin, $\left.t_{(8)}=4.3, p=0.003\right)$, except when rats received crossed unilateral infusions of anisomycin into PRh

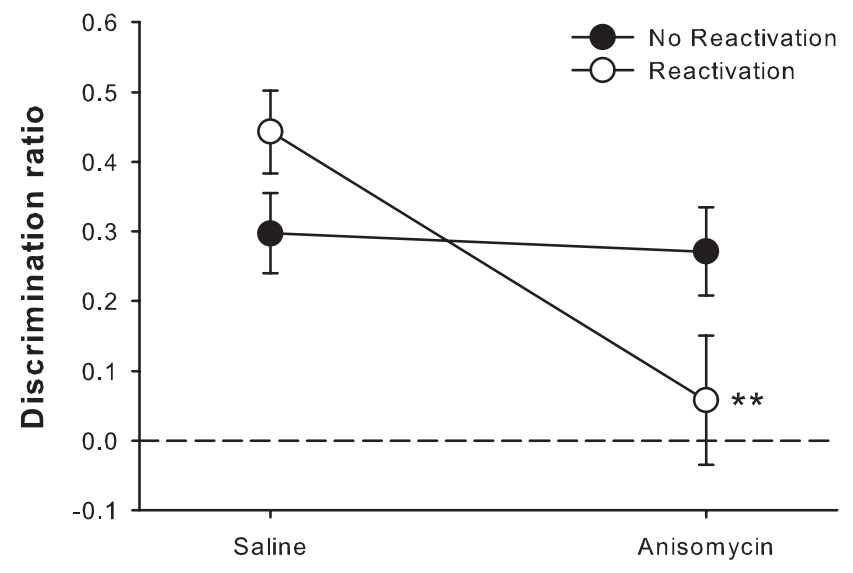

Treatment

Figure 8. Spontaneous object recognition performance of rats in Experiment 7. Crossed unilateral administration of anisomycin into PRh and HPC immediately following memory reactivation in the presence of a novel contextual cue prevented reconsolidation of the sample object trace. Inhibition of protein synthesis did not affect object recognition memory in rats that did not undergo a memory reactivation phase before drug delivery. Data are presented as average discrimination ratio ( \pm SEM) from the choice phase administered $24 \mathrm{~h}$ after drug infusions. ${ }^{* *} p<0.01$ (anisomycin vs saline in reactivation group).

and HPC immediately after the reactivation phase $\left(t_{(9)}=\right.$ 0.623, $p=0.549)$.

Analysis of general exploration values indicated no significant differences between conditions in terms of total exploration levels (sample: drug, $F_{(1,17)}=0.005, p=0.946$, group, $F_{(1,17)}=0.143, p=$ 0.71 , drug $\times$ group, $F_{(1,17)}=0.447, p=0.513$; reactivation: $t_{(9)}=$ $0.161, p=0.876$; choice: drug, $F_{(1,17)}=0.366, p=0.553$, group, $F_{(1,17)}=0.042, p=0.841$, drug $\times$ group, $\left.F_{(1,17)}=2.144, p=0.161\right)$ or phase duration (sample: drug, $F_{(1,17)}=0.032, p=0.861$, group, $F_{(1,17)}=0.093, p=0.764$, drug $\times$ group, $F_{(1,17)}=0.456, p=0.509$; reactivation: $\left.t_{(9)}=0.784, p=0.453\right)$.

\section{Discussion}

These data support previous findings reporting memory reconsolidation in the SOR task (Bozon et al., 2003; Kelly et al., 2003; Akirav and Maroun, 2006; Rossato et al., 2007; Maroun and Akirav, 2008; Lima et al., 2009; Winters et al., 2009; Davis et al., 2010; Romero-Granados et al., 2010) and provide new insight into the dynamic nature of object memory storage and retrieval.

These results represent the first demonstration of an important role for $\mathrm{PRh}$ in object memory reconsolidation. In contrast to hippocampal involvement, PRh appears to contribute to object memory reconsolidation under a variety of memory reactivation conditions; this pattern of prevalent involvement in object memory reconsolidation is consistent with current perspectives that regard $\mathrm{PRh}$ as central to information processing for object representation (Buckley and Gaffan, 2006; Bussey and Saksida, 2007; Murray et al., 2007), as well as past findings implicating $\mathrm{PRh}$ in object memory acquisition, consolidation, and retrieval (Winters et al., 2008).

These data also provide insight into the specific role of the $\mathrm{HPC}$ in object memory processing. Unlike PRh, intra-HPC anisomycin impaired object memory only when memory reactivation occurred in the presence of novel contextual information. This finding is consistent with past results showing impaired ability of HPC-lesioned rats to recognize familiar objects in novel contexts (O'Brien et al., 2006; Piterkin et al., 2008) and more generally with previous suggestions that the HPC may only be- 
come involved in object recognition performance when contextual information is highly relevant (Gaffan, 1994; Cassaday and Rawlins, 1997; Zola et al., 2000; Bussey and Aggleton, 2002; Mumby et al., 2002; Winters et al., 2004; Aggleton and Brown, 2005; Forwood et al., 2005).

The selective nature of object memory reconsolidation blockade following intra-HPC anisomycin conflicts with past findings suggesting a role for the HPC in updating object memory traces when novel objects are present (Rossato et al., 2007). The current results are consistent with an updating function, but only when the novel information is contextual in nature. The disparity between the present findings and those reported by Rossato et al. (2007) may be related to procedural differences. First, Rossato et al. (2007) infused $160 \mu \mathrm{g}$ of anisomycin into the dorsal HPC compared with our dose of $100 \mu$ g per hemisphere. However, this lower dose was sufficient to produce large deficits in each of the $\mathrm{PRh}$ experiments in the current study and caused significant memory impairment when administered into the HPC following the context change reactivation. Thus, it seems unlikely that the absence of impairment by intra-HPC anisomycin in the novel object reactivation condition can be explained by our lower drug dose.

A second potential explanation involves the difference between object presentations in the two studies. Unlike in the current study, Rossato et al. (2007) presented two different objects in the sample phase. This, combined with subsequent presentation of yet another object in the reactivation phase might encourage encoding of HPC-dependent relational information. Hippocampal involvement could have been further promoted by the use of an open field to assess SOR in the Rossato et al. (2007) study. There are several reports of hippocampal effects on SOR when tested in an open field (Clark et al., 2000; Broadbent et al., 2004), and such effects have been ascribed to the potential involvement of spatial or contextual factors that may be less influential when the SOR task is run in the Y-shaped apparatus (Winters et al., 2004; Forwood et al., 2005). Despite their differences, both Rossato et al. (2007) and the current study imply a specific role for the HPC in object memory reconsolidation when salient novel contextual or relational information is present during reactivation. This finding has important implications, not only for hippocampal involvement, but more broadly in terms of the dynamics of object memory storage and updating.

The current results imply a substantial change in the organization of the object memory substrate following reactivation in the presence of novel contextual information, because anisomycin infusion into either PRh or the HPC following such a reactivation appeared to abolish the object memory, as did pharmacological disconnection of hippocampal and perirhinal functions. This contrasts with the inconsequential effects of intra-HPC anisomycin in the other memory reactivation conditions. Recent research has supported a "transformation hypothesis," which suggests that memories can become less context-specific over time and that such changes could explain the differential dependence of certain remote memories on the HPC (Winocur et al., 2007, 2009; Wiltgen et al., 2010). The present results could reflect a reversal of this scenario. That is, the encoding of the sample object occurred in an habituated environment that had been specifically designed to lack notable contextual features. Inclusion of the textured floor insert, however, during the reactivation phase produced a salient contextual change which prompted greater involvement of the HPC for reconsolidation of the object memory. As such, just as some memories can become less context- and HPC-dependent, presentation of salient con- textual information during sensitive periods might render memories more reliant on the HPC than when first encoded.

What is particularly intriguing about this apparent memory trace reorganization is the fact that the object memory becomes reliant on the HPC while retaining its PRh dependence; indeed, the results of the final experiment indicate that object memory reconsolidation following reactivation in an altered context requires functional interaction between the HPC and PRh. Accordingly, previous research has suggested that the HPC and PRh interact when contextual and object information must be integrated (Gaffan and Parker, 1996; Bussey et al., 2000, 2001; Bussey and Aggleton, 2002; Winters et al., 2004; Jo and Lee, 2010). The current results, however, raise questions about the specific nature of hippocampal involvement in the object memory reconsolidation process. Results from other memory paradigms suggest that addition of new information to an established memory trace depends on consolidation processes, while restabilization of the original information occurs via distinct reconsolidation processes (Tronel et al., 2005; Debiec et al., 2006; Alberini, 2011). In the present study, however, intra-HPC or intra-PRh anisomycin appeared to abolish object recognition memory when applied immediately after reactivation in the altered context. This would not be expected if the HPC was merely mediating the addition of novel contextual information to the original object memory. On the contrary, the present results suggest a substantial rearrangement of the circuitry required for successful object recognition. Future studies should address this intriguing implication to determine whether other types of memory are subject to the same kind of circuit level reorganization implied by the present findings.

Previous studies using other behavioral paradigms have also shown that post-reactivation reconsolidation can strengthen associations (Lee, 2008; Alberini, 2011). The present data, however, are not fully consistent with these findings. Although in four of the seven experiments discrimination ratios were numerically higher in the saline-treated rats undergoing reactivation than in the no reactivation condition, these tended to be marginal differences. Some have argued, however, that the SOR task cannot be used to infer degrees of object memory strength, but merely whether animals can significantly discriminate between the novel and sample objects (Gaskin et al., 2010). If this is the case, then additional measures will be required to assess the potential impact of memory reactivation episodes on the strength of object memories.

An important final consideration concerns the specific mechanism of amnesic effects caused by intracranial anisomycin. While such effects are commonly attributed to protein synthesis inhibition, anisomycin and other protein synthesis inhibitors (PSIs) can influence numerous biochemical processes. For instance, PSIs can inhibit monoamine function (Flexner and Goodman, 1975; Canal et al., 2007), influence post-translational modification of proteins, including MAPK and CREB (Routtenberg and Rekart, 2005), which are important components of memory-related biochemical cascades, and cause apoptosis at doses below those responsible for protein synthesis inhibition (Iordanov et al., 1997; Rudy et al., 2006). Apoptotic effects cannot fully explain the impairments observed in the present withinsubjects study, as there was no reduction in vehicle condition performance by rats treated with anisomycin on previous trials. Nonetheless, other physiological effects of anisomycin should not be discounted when considering the specific mechanism responsible for its amnesic effects.

The present results highlight the fact that long-term memory storage is an ongoing, dynamic process. A significant implication 
of these findings is that the synapses responsible for a memory trace can, under certain conditions, become uncoupled to such an extent that successful retention of the original information depends on interaction between multiple brain regions, some of which may not have been critically involved in the original storage of the memory. The potential of reactivation events to exert such significant impact on the organization of the memory trace is consistent with recent suggestions that "reconsolidation" processes represent essential aspects of a prolonged, or "lingering," consolidation period during which the original memory trace remains open to modification (Dudai and Eisenberg, 2004; Alberini, 2005). Better understanding of the conditions mediating perirhinal-hippocampal interaction in object memory reconsolidation and the apparent memory trace reorganization shown here will shed further light on the dynamic nature of memory storage, retrieval, and updating processes in the mammalian brain.

\section{References}

Aggleton JP, Brown MW (2005) Contrasting hippocampal and perirhinal cortex function using immediate early gene imaging. Q J Exp Psychol B $58: 218-233$

Akirav I, Maroun M (2006) Ventromedial prefrontal cortex is obligatory for consolidation and reconsolidation of object recognition memory. Cereb Cortex 16:1759-1765.

Alberini CM (2005) Mechanisms of memory stabilization: are consolidation and reconsolidation similar or distinct processes? Trends Neurosci 28:51-56.

Alberini CM (2011) The role of reconsolidation and the dynamic process of long-term memory formation and storage. Front Behav Neurosci 5:12.

Barker GR, Bashir ZI, Brown MW, Warburton EC (2006a) A temporally distinct role for group I and group II metabotropic glutamate receptors in object recognition memory. Learn Mem 13:178-186.

Barker GR, Warburton EC, Koder T, Dolman NP, More JC, Aggleton JP, Bashir ZI, Auberson YP, Jane DE, Brown MW (2006b) The different effects on recognition memory of perirhinal kainate and NMDA glutamate receptor antagonism: implications for underlying plasticity mechanisms. J Neurosci 26:3561-3566.

Barker GR, Bird F, Alexander V, Warburton EC (2007) Recognition memory for objects, place, and temporal order: a disconnection analysis of the role of the medial prefrontal cortex and perirhinal cortex. J Neurosci 27:2948-2957.

Bartko SJ, Winters BD, Cowell RA, Saksida LM, Bussey TJ (2007) Perceptual functions of perirhinal cortex in rats: zero-delay object recognition and simultaneous oddity discriminations. J Neurosci 27:2548-2559.

Berman DE, Hazvi S, Neduva V, Dudai Y (2000) The role of identified neurotransmitter systems in the response of insular cortex to unfamiliar taste: activation of ERK1-2 and formation of a memory trace. J Neurosci 20:7017-7023.

Bozon B, Davis S, Laroche S (2003) A requirement for the immediate early gene zif268 in reconsolidation of recognition memory after retrieval. Neuron 40:695-701.

Broadbent NJ, Squire LR, Clark RE (2004) Spatial memory, recognition memory, and the hippocampus. Proc Natl Acad Sci USA 101:14515-14520.

Buckley MJ, Gaffan D (2006) Perirhinal cortical contributions to object perception. Trends Cogn Sci 10:100-107.

Burwell RD (2001) Borders and cytoarchitecture of the perirhinal and postrhinal cortices in the rat. J Comp Neurol 437:17-41.

Bussey TJ, Aggleton JP (2002) The "what" and "where" of event memory: independence and interactivity within the medial temporal lobe. In: The cognitive neuroscience of memory: encoding and retrieval (Parker A, Wilding E, Bussey TJ, eds), pp 217-233. London: Psychology.

Bussey TJ, Saksida LM (2007) Memory, perception, and the ventral visualperirhinal-hippocampal stream: thinking outside of the boxes. Hippocampus 17:898-908.

Bussey TJ, Duck J, Muir JL, Aggleton JP (2000) Distinct patterns of behavioural impairments resulting from fornix transection or neurotoxic lesions of the perirhinal and postrhinal cortices in the rat. Behav Brain Res 111:187-202.
Bussey TJ, Dias R, Amin E, Muir JL, Aggleton JP (2001) Perirhinal cortex and place-object conditional learning in the rat. Behav Neurosci 115:776-785.

Canal CE, Chang Q, Gold PE (2007) Amnesia produced by altered release of neurotransmitters after intraamygdala injections of a protein synthesis inhibitor. Proc Natl Acad Sci U S A 104:12500-12505.

Cassaday HJ, Rawlins JN (1997) The hippocampus, objects, and their contexts. Behav Neurosci 111:1228-1244.

Clark RE, Zola SM, Squire LR (2000) Impaired recognition memory in rats after damage to the hippocampus. J Neurosci 20:8853-8860.

Davis S, Renaudineau S, Poirier R, Poucet B, Save E, Laroche S (2010) The formation and stability of recognition memory: what happens upon recall? Front Behav Neurosci 4:177.

Debiec J, Doyère V, Nader K, Ledoux JE (2006) Directly reactivated, but not indirectly reactivated, memories undergo reconsolidation in the amygdala. Proc Natl Acad Sci U S A 103:3428-3433.

Dix SL, Aggleton JP (1999) Extending the spontaneous preference test of recognition: evidence of object-location and object-context recognition. Behav Brain Res 99:191-200.

Dudai Y (2004) The neurobiology of consolidations, or, how stable is the engram? Annu Rev Psychol 55:51-86.

Dudai Y, Eisenberg M (2004) Rites of passage of the engram: reconsolidation and the lingering consolidation hypothesis. Neuron 44:93-100.

Flexner LB, Goodman RH (1975) Studies on memory: inhibitors of protein synthesis also inhibit catecholamine synthesis. Proc Natl Acad Sci U S A 72:4660-4663

Forwood SE, Winters BD, Bussey TJ (2005) Hippocampal lesions that abolish spatial maze performance spare object recognition memory at delays of up to 48 hours. Hippocampus 15:347-355.

Gaffan D (1994) Dissociated effects of perirhinal cortex ablation, fornix transection and amygdalectomy: evidence for multiple memory systems in the primate temporal lobe. Exp Brain Res 99:411-422.

Gaffan D, Parker A (1996) Interaction of perirhinal cortex with the fornixfimbria: memory for objects and "object-in-place" memory. J Neurosci 16:5864-5869.

Gaskin S, Tardif M, Cole E, Piterkin P, Kayello L, Mumby DG (2010) Object familiarization and novel-object preference in rats. Behav Processes 83:61-71.

Hardt O, Einarsson EO, Nader K (2010) A bridge over troubled water: reconsolidation as a link between cognitive and neuroscientific memory research traditions. Annu Rev Psychol 61:141-167.

Hupbach A, Gomez R, Hardt O, Nadel L (2007) Reconsolidation of episodic memories: a subtle reminder triggers integration of new information. Learn Mem 14:47-53.

Inda MC, Muravieva EV, Alberini CM (2011) Memory retrieval and the passage of time: from reconsolidation and strengthening to extinction. J Neurosci 31:1635-1643.

Iordanov MS, Pribnow D, Magun JL, Dinh TH, Pearson JA, Chen SL, Magun BE (1997) Ribotoxic stress response: activation of the stress-activated protein kinase JNK1 by inhibitors of the peptidyl transferase reaction and by sequence-specific RNA damage to the alpha-sarcin/ricin loop in the 28S rRNA. Mol Cell Biol 17:3373-3381.

Jo YS, Lee I (2010) Disconnection of the hippocampal-perirhinal cortical circuits severely disrupts object-place paired associative memory. J Neurosci 30:9850-9858.

Kelly A, Laroche S, Davis S (2003) Activation of mitogen-activated protein kinase/extracellular signal-regulated kinase in hippocampal circuitry is required for consolidation and reconsolidation of recognition memory. J Neurosci 23:5354-5360.

Lee JL (2008) Memory reconsolidation mediates the strengthening of memories by additional learning. Nat Neurosci 11:1264-1266.

Lee JL (2010) Memory reconsolidation mediates the updating of hippocampal memory content. Front Behav Neurosci 4:168.

Lima RH, Rossato JI, Furini CR, Bevilaqua LR, Izquierdo I, Cammarota M (2009) Infusion of protein synthesis inhibitors in the entorhinal cortex blocks consolidation but not reconsolidation of object recognition memory. Neurobiol Learn Mem 91:466-472.

Lukowiak K, Fras M, Smyth K, Wong C, Hittel K (2007) Reconsolidation and memory infidelity in Lymnaea. Neurobiol Learn Mem 87:547-560.

Maroun M, Akirav I (2008) Arousal and stress effects on consolidation and reconsolidation of recognition memory. Neuropsychopharmacology 33:394-405. 
Martin JH (1991) Autoradiographic estimation of the extent of reversible inactivation produced by microinjection of lidocaine and muscimol in the rat. Neurosci Lett 127:160-164.

McGaugh JL (2000) Memory—a century of consolidation. Science 287: $248-251$.

McTighe SM, Cowell RA, Winters BD, Bussey TJ, Saksida LM (2010) Paradoxical false memory for objects after brain damage. Science 330 : $1408-1410$

Misanin JR, Miller RR, Lewis DJ (1968) Retrograde amnesia produced by electroconvulsive shock after reactivation of a consolidated memory trace. Science 160:554-555.

Morris RG, Inglis J, Ainge JA, Olverman HJ, Tulloch J, Dudai Y, Kelly PA (2006) Memory reconsolidation: sensitivity of spatial memory to inhibition of protein synthesis in dorsal hippocampus during encoding and retrieval. Neuron 50:479-489.

Mumby DG, Gaskin S, Glenn MJ, Schramek TE, Lehmann H (2002) Hippocampal damage and exploratory preferences in rats: memory for objects, places, and contexts. Learn Mem 9:49-57.

Murray EA, Bussey TJ, Saksida LM (2007) Visual perception and memory: a new view of medial temporal lobe function in primates and rodents. Annu Rev Neurosci 30:99-122.

Nader K (2003) Memory traces unbound. Trends Neurosci 26:65-72.

O’Brien N, Lehmann H, Lecluse V, Mumby DG (2006) Enhanced contextdependency of object recognition in rats with hippocampal lesions. Behav Brain Res 170:156-162.

Paxinos G, Watson C (1998) The rat brain in stereotaxic coordinates, Ed 3. London: Academic.

Piterkin P, Cole E, Cossette MP, Gaskin S, Mumby DG (2008) A limited role for the hippocampus in the modulation of novel-object preference by contextual cues. Learn Mem 15:785-791.

Rodriguez-Ortiz CJ, De la Cruz V, Gutiérrez R, Bermudez-Rattoni F (2005) Protein synthesis underlies post-retrieval memory consolidation to a restricted degree only when updated information is obtained. Learn Mem 12:533-537.

Rodriguez-Ortiz CJ, Garcia-DeLaTorre P, Benavidez E, Ballesteros MA, Bermudez-Rattoni F (2008) Intrahippocampal anisomycin infusions disrupt previously consolidated spatial memory only when memory is updated. Neurobiol Learn Mem 89:352-359.

Romero-Granados R, Fontán-Lozano A, Delgado-García JM, Carrión AM (2010) From learning to forgetting: behavioral, circuitry, and molecular properties define the different functional states of the recognition memory trace. Hippocampus 20:584-595.

Rosenblum K, Meiri N, Dudai Y (1993) Taste memory: the role of protein synthesis in gustatory cortex. Behav Neural Biol 59:49-56.
Rossato JI, Bevilaqua LR, Myskiw JC, Medina JH, Izquierdo I, Cammarota M (2007) On the role of hippocampal protein synthesis in the consolidation and reconsolidation of object recognition memory. Learn Mem 14:36-46.

Routtenberg A, Rekart JL (2005) Post-translational protein modification as the substrate for long-lasting memory. Trends Neurosci 28:12-19.

Rudy JW, Biedenkapp JC, Moineau J, Bolding K (2006) Anisomycin and the reconsolidation hypothesis. Learn Mem 13:1-3.

Sara SJ (2000) Retrieval and reconsolidation: toward a neurobiology of remembering. Learn Mem 7:73-84.

Tronel S, Milekic MH, Alberini CM (2005) Linking new information to a reactivated memory requires consolidation and not reconsolidation mechanisms. PLoS Biol 3:e293.

Wiltgen BJ, Zhou M, Cai Y, Balaji J, Karlsson MG, Parivash SN, Li W, Silva AJ (2010) The hippocampus plays a selective role in the retrieval of detailed contextual memories. Curr Biol 20:1336-1344.

Winocur G, Moscovitch M, Sekeres M (2007) Memory consolidation or transformation: context manipulation and hippocampal representations of memory. Nat Neurosci 10:555-557.

Winocur G, Frankland PW, Sekeres M, Fogel S, Moscovitch M (2009) Changes in context-specificity during memory reconsolidation: selective effects of hippocampal lesions. Learn Mem 16:722-729.

Winters BD, Bussey TJ (2005a) Glutamate receptors in perirhinal cortex mediate encoding, retrieval, and consolidation of object recognition memory. J Neurosci 25:4243-4251.

Winters BD, Bussey TJ (2005b) Transient inactivation of perirhinal cortex disrupts encoding, retrieval, and consolidation of object recognition memory. J Neurosci 25:52-61.

Winters BD, Forwood SE, Cowell RA, Saksida LM, Bussey TJ (2004) Double dissociation between the effects of peri-postrhinal cortex and hippocampal lesions on tests of object recognition and spatial memory: heterogeneity of function within the temporal lobe. J Neurosci 24:5901-5908.

Winters BD, Saksida LM, Bussey TJ (2008) Object recognition memory: neurobiological mechanisms of encoding, consolidation and retrieval. Neurosci Biobehav Rev 32:1055-1070.

Winters BD, Tucci MC, DaCosta-Furtado M (2009) Older and stronger object memories are selectively destabilized by reactivation in the presence of new information. Learn Mem 16:545-553.

Winters BD, Bartko SJ, Saksida LM, Bussey TJ (2010) Muscimol, AP5, or scopolamine infused into perirhinal cortex impairs two-choice visual discrimination learning in rats. Neurobiol Learn Mem 93:221-228.

Zola SM, Squire LR, Teng E, Stefanacci L, Buffalo EA, Clark RE (2000) Impaired recognition memory in monkeys after damage limited to the hippocampal region. J Neurosci 20:451-463. 